\title{
Cloning and expression analysis of the 37-kDa laminin receptor precursor gene from Hyriopsis cumingii
}

X.Z. Chang', J.L. Li ${ }^{1,2}$, Z.Y. Bai ${ }^{1}$ and X.L. Li ${ }^{1}$

${ }^{1}$ Key Laboratory of Freshwater Fishery Genetic Resources, Ministry of Agriculture, Shanghai Ocean University, Shanghai, China

${ }^{2}$ Aquaculture Division, E-Institute of Shanghai Universities, College of Fisheries and Life Science, Shanghai Ocean University, Shanghai, China

Corresponding author: J.L. Li

E-mail: j1li2009@126.com

Genet. Mol. Res. 12 (4): 6130-6139 (2013)

Received July 18, 2013

Accepted October 10, 2013

Published December 2, 2013

DOI http://dx.doi.org/10.4238/2013.December.2.10

ABSTRACT. Hyriopsis cumingii is an economically important
freshwater pearl mussel with high pearl quality that is endemic in
China. Investigation of genes relevant to shell formation is important
for increased pearl output. The substances that form mollusk shells are
secreted by epithelial cells in the mantle, the proliferation of which
influences secretion ability. This study focused on the proliferation-
related $37-\mathrm{kDa}$ laminin receptor precursor ( $37 L R P$ ) of $H$. cumingii. The
full-length cDNA ( $1133 \mathrm{bp}$ ) encoding this 300 -amino acid protein was
cloned from the mantle. Quantitative fluorescence analysis showed that
$37 L R P$ expressed in eight tissues, with the highest expression observed
in the liver, and its expression pattern in the mantle reflected shell repair.
During repair, $37 L R P$ expression was higher in the experimental shell
repair group than that in the control group, exhibiting an initial increase
followed by a decrease in expression, and returning to basal levels on
completion of the repair. A similar trend was also observed with respect 
to immunity and cellular metabolism. Expression of the 37LRP protein in the experimental group was significantly higher than that in the control group at the first and second days after shell injury. After 4 days, $37 L R P$ expression in the experimental group was lower than that in the control group. In situ hybridization revealed a strong positive signal corresponding to the 37LRP mRNA at the horny grooves of the mantle, evagination, and in epithelial cells of the velum, which implicated these areas in the repair and formation of the cuticle, prismatic layer, and nacre.

Key words: Hyriopsis cumingii; Laminin receptor precursor; Expression analysis; In situ hybridization; Shell repair

\section{INTRODUCTION}

It is widely acknowledged that the substances forming mollusk shells are secreted by the epithelial cells in the mantle (McKenna et al., 2001), and that the shell is formed by ordered deposition of $\mathrm{CaCO}_{3}$ crystals under the regulation of a protein matrix also secreted by the epithelial cells in the mantle (Donaldson et al., 2000). Fu et al. (2008) analyzed the $37-\mathrm{kDa}$ laminin receptor precursor gene (37LRP) of Pinctada fucata and speculated that the encoded protein was involved in mantle epithelial cell proliferation and nacre secretion (Livak and Schmittgen, 2001). The 37LRP, which is the precursor of the $67-\mathrm{kDa}$ laminin receptor (LR), has two high-affinity laminin (LN) binding sites (Malinda et al., 1999): the G peptide (Castronovo et al., 1991; Taraboletti et al., 1993; Menard et al., 1997), and another, located at the C-terminal (Magnifico et al., 1996; Satoh et al., 1999). The 67-kDa LR also has a strong binding affinity for LN, which is an important non-integrated LR used as a marker for tumor detection, is important in cell proliferation and migration, and combines with various extracellular signals, such as extracellular matrix, viruses, and bacteria (Vacca et al., 1993; Sanjuan et al., 1996; Bai et al., 2009; Li et al., 2010). Based on its important role in cell proliferation, and the differences in the survival environments of marine and freshwater mussels, as well as the crystal form of nacre and prismatic layers, we studied the $37 L R P$ gene of Hyriopsis cumingii. This is the first report on $37 L R P$ in a freshwater mussel.

The $37 L R P$ cDNA was first cloned from the mantle of $H$. cumingii and then sequenced. Its expression during shell repair was analyzed by real-time polymerase chain reaction (PCR) to verify its function. The shell is usually made up of three layers: the corneum, the prismatic layer, and the nacreous layer. The outermost corneum (horny layer) is formed by secretion from horny groove cells between the middle and outer pleats. The internal prismatic layer is formed by secretion from epithelial cells in the mantle near the ventral region. The nacre layer is formed from secretion of epithelial cells in the mantle near the dorsal region (Suzuki et al., 1991; Kibbey et al., 1992; Awaji and Suzuki, 1995; Miyamoto et al., 1996; Nomizu et al., 1998; Coggin et al., 1999). In situ hybridization was performed to investigate the function of this gene in shell repair.

\section{MATERIAL AND METHODS}

\section{Material and RNA isolation}

Two-year-old H. cumingii were obtained from the Shanghai Aquaculture Engineering 
Center (coastal). According to the requirements of the experiments, the mantle, blood, gill, foot, liver, kidney, intestine, and adductor muscle were isolated, snap-frozen in liquid nitrogen, and stored at $-80^{\circ} \mathrm{C}$. Total RNA was extracted from the mantle, blood, gill, foot, liver, kidney, intestine, and adductor muscle using Trizol according to manufacturer instructions (TaKaRa, Dalian, China).

\section{Amplification, cloning, and sequencing of $37 L R P$ cDNA}

Primers of 5'- and 3'-rapid amplification of cDNA ends (RACE) were designed using Primer Premier 5.0 software and the BLAST program according to the labeled expressed sequence tags in the constructed mantle cDNA library (Ardini et al., 1998) and synthesized (Sangon, Shanghai, China). The 3'-end was amplified using the TaKaRa RNA PCR Kit (AMV) Ver. 3.0 (TaKaRa) with the upstream primer L-F (5'-CCGTTCTCAAGTTTGCCTCC-3') and the downstream primer (M13 Primer kit) M4 (5'-GTTTTCCCAGTCACGAC-3'). The 5'-end was amplified using the SMART ${ }^{\mathrm{TM}}$ RACE cDNA Amplification Kit (BD) and the Advantage ${ }^{\circledR}$ 2PCR Enzyme System kit (Clontech, Santa Clara, CA, USA). The downstream primer (LR), designed using Primer Premier 5.0, was 5'-GACGAAGCACCTCCCTTGCCAGC-3'. The upstream primer from the kit was 5'-CTAATACGACTCACTATAGGGCAAGCAGTGGTAT CAACGCAGAGT-3' (long); 5'-CTAATACGACTCACTATAGGGC-3' (short). The RACEamplified products were extracted and separated by $1.5 \%$ agarose gel electrophoresis. The PCR product of the target gene was purified using the TIANgel Midi Purification Kit (TIANGEN, Beijing, China). The recombinant plasmid was obtained by ligating the purified RACE products with the pMD19-T vector $(\mathrm{TaKaRa})$. The resulting construct was transformed into competent Escherichia coli DH5 $\alpha$ cells and cultured on Luria-Bertani plates [containing ampicillin, isopropyl-b-D-1-thiogalactopyranoside (IPTG), and X-gal]. Blue/white screening was used to detect the inserted fragment. The positive clone obtained was sequenced (Shanghai Branch, Beijing Luhe, China).

\section{Sequencing}

The similarity of the nucleotide and amino acid sequences was analyzed using the BLAST database (http://www.ncbi.nlm.nih.gov/BLAST). The correct open reading frame (ORF) was determined using the ORF Finder program (http://www.ncbi.nlm.nih.gov/projects/ gorf/) (Gloe et al., 1999). The physical parameters of the amino acid sequences were predicted using ProtParam and SignalP 4.0 servers. Multiple comparisons of the amino acid and coding nucleotide sequences were performed using ClustalW1.8 and BioEdit softwares.

\section{Quantitative real-time PCR (qPCR) analysis of $37 L R P$ expression in eight tissues}

Total RNA was extracted from the blood, gill, mantle, foot, adductor muscle, liver, kidney, and intestine as previously described. qPCR analysis of $37 L R P$ expression was conducted using the following primers designed using Primer Premier 5.0: 37LRP, upstream primer LrtF: 5'-TCACCTGGGAGCCACAAA-3' and downstream primer Lrt-R: 5'-GCAGAGATGAC GCAAACATC-3'; $\beta$-actin-F: 5'-ACGGATAACACAAGGAAAGGAAAC-3' and $\beta$-actin-R: 5'-ATGGATGGAAACACGGCTCT-3'. $\beta$-actin expression in $H$. cumingii was analyzed as the internal reference. Each sample was analyzed in triplicate using the iScript ${ }^{\mathrm{TM}} \mathrm{cDNA}$ Synthesis 
Kit and $\mathrm{iQ}^{\mathrm{TM}} \mathrm{SYBR}^{\circledR}$ Green Supermix (BIO-RAD, USA) in a $20-\mu \mathrm{L}$ reaction system containing $10 \mu \mathrm{L}$ iQTM SYBR ${ }^{\circledR}$ Green Supermix, $0.8 \mu \mathrm{L} 10 \mathrm{M}$ primers, $6.8 \mu \mathrm{L}$ RNase-free water, and $1.6 \mu \mathrm{L}$ cDNA. Amplification was performed using a BIO-RAD CFX $96^{\mathrm{TM}}$ under the following conditions: $95^{\circ} \mathrm{C}$ (pre-denaturation) for $30 \mathrm{~s}, 95^{\circ} \mathrm{C}$ (degeneration) for $5 \mathrm{~s}$, and $60^{\circ} \mathrm{C}$ (annealing) for $30 \mathrm{~s}$ ( 40 cycles) with a temperature gradient of $0.5^{\circ} \mathrm{C}$ per cycle from $65^{\circ}$ to $95^{\circ} \mathrm{C}$. The relative expression of the target gene and reference gene was calculated using the $2^{-\Delta \Delta \mathrm{Ct}}$ method (Landowski et al., 1995) and analyzed with the SigmaPlot10.0 software.

\section{qPCR analysis of $37 L R P$ expression during shell repair}

Healthy adult $H$. cumingii $(\mathrm{N}=60)$ of similar shape were randomly and equally divided into the experimental shell repair and control groups. The shell of $H$. cumingii in the experimental group was damaged (a V-shaped gap near the edge of the adductor muscle was formed) and randomly divided into 10 groups ( $\mathrm{N}=3$ per group). The control group was also divided into 10 groups. $H$. cumingii from the experimental and control groups $(\mathrm{N}=3$ from each group for each time point analyzed during the shell repair process) were placed together in a mesh bag, suspended, and maintained at the Shanghai Aquaculture Engineering Center (Binhai). The mantle and liver were collected at specific time points after shell damage $(2,6$, $12,24 \mathrm{~h}$, and 2, 4, 7, 15, and 30 days). Tissues were snap-frozen in liquid nitrogen and stored at $-80^{\circ} \mathrm{C}$. Total RNA was extracted for qPCR analysis of $37 L R P$ expression during shell repair.

\section{In situ hybridization of $37 L R P$ in the mantle}

The mantle was collected from living $H$. cumingii, fixed in 4\% paraformaldehyde for $24 \mathrm{~h}$, rinsed with 1X phosphate-buffered saline (prepared with diethylpyrocarbonate-treated water), dried, fixed with methanol, and stored at $-20^{\circ} \mathrm{C}$. The fixed tissues were dehydrated, embedded in paraffin, and sectioned for evaluation. For hybridization, sections were incubated with hybridization solution and probe at $55^{\circ} \mathrm{C}$ overnight and then incubated with NBT-BCIP in the dark for $12 \mathrm{~h}$. Sections were dehydrated, mounted, and photographed. The DIG RNA Labeling Kit (SP6/P7) and DIG Nucleic Acid Detection Kit (Roche, Mannheim, Germany) were used for the synthesis and detection of the digoxin-labeled RNA probe, respectively.

\section{RESULTS}

\section{cDNA and amino acid sequence of $37 L R P$ and homology analysis}

The full-length cDNA sequence (1133 bp) of the 37LRP (accession No. JX441866) was obtained after amplification and found to comprise a 5'-untranslated region (UTR) of 71 bp, a 3'-UTR of $159 \mathrm{bp}$, and an ORF of $903 \mathrm{bp}$, encoding 300-amino acid residues (Figure 1). The total atomic weight was 4625 and the molecular formula was $\mathrm{C}_{1477} \mathrm{H}_{2291} \mathrm{~N}_{395} \mathrm{O}_{452} \mathrm{~S}_{10}$ (molecular weight was approximately $33.1 \mathrm{kDa}$ ). Comparisons of the full-length $\mathrm{H}$. cumingii 37LRP protein with that of other species using the ClustalW software (Figure 2) showed a high degree of similarity. The amino acid sequence of $H$. cumingii 37LRP exhibited the highest similarity (80\%) with those of P. fucata, Bos taurus, Chlorocebus aethiops, and Rattus norvegicus (73\%), and with Homo sapiens and Xenopus laevis (72\%). 


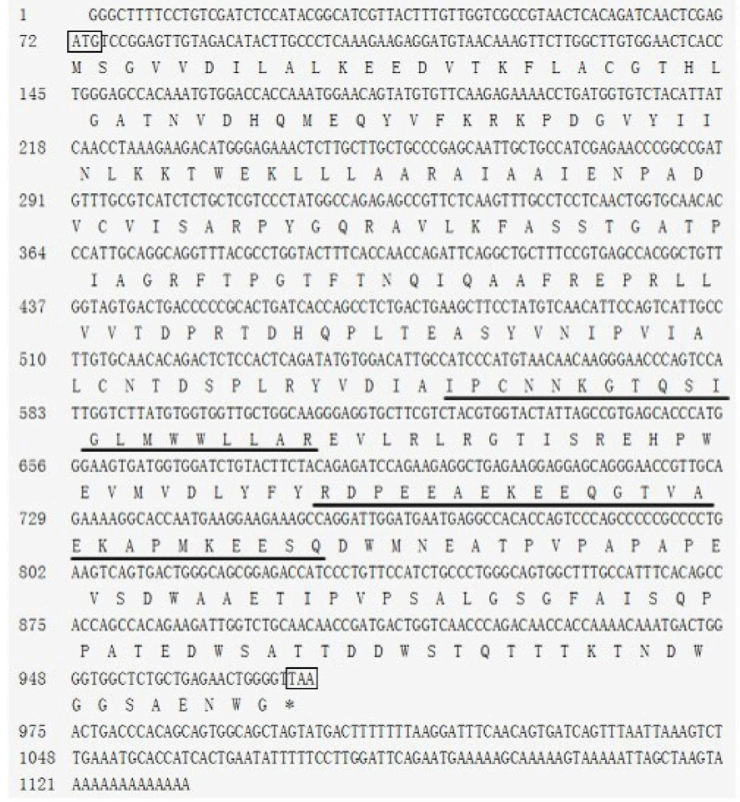

Figure 1. Full-length cDNA and deduced amino acid sequences of $37 L R P$ from Hyriopsis cumingii. Box: initiator codon and stop codon; underlined: laminin-binding sites.

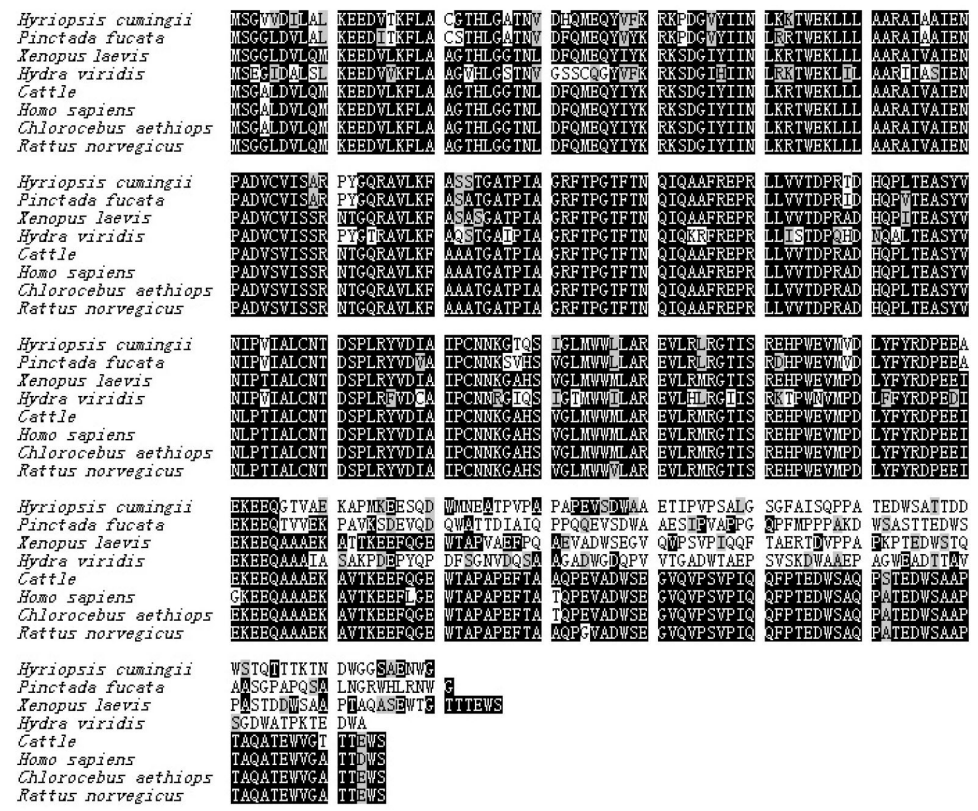

Figure 2. Comparison of amino acid sequences at the putative LN binding sites in the 37LRPs among Hyriopsis cumingii and other species. The black line above the acid sequences shows the two LN binding sites in 37LRPs. 


\section{Expression of $37 L R P$ in eight tissues in $H$. cumingii}

Quantitative PCR analysis showed that $37 L R P$ was expressed in the mantle, blood, gill, foot, liver, kidney, intestine, and adductor muscle tissues. Highest expression was detected in the liver, lowest in the blood, and similarly low levels in all other tissues evaluated (Figure 3).

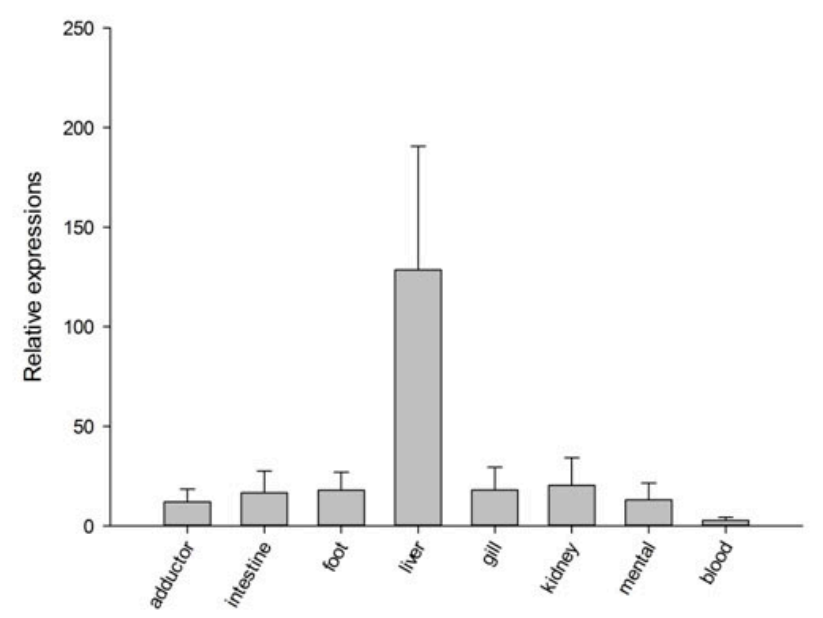

Figure 3. Tissue distribution of 37LRP mRNA in Hyriopsis cumingii.

\section{Expression of $37 L R P$ in the liver and mantle during shell repair}

During shell repair, expression of $37 L R P$ in the liver was initially increased, followed by decreased expression and a subsequent increase. Expression in the experimental group was significantly higher than that in the control group during the first and second days after shell damage, and was lower than that in the control group after four days (Figure 4).

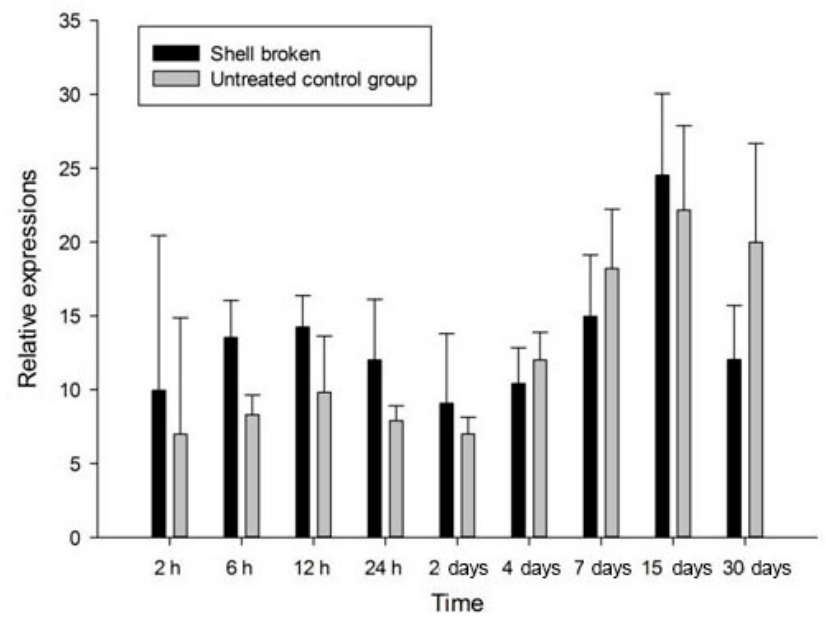

Figure 4. Relative expression of $37 L R P$ in liver after shell damage at each sampling time point. 
Expression of $37 L R P$ in the mantle initially increased and subsequently decreased during shell repair. Its expression in the experimental group was higher than that in the control group at each time point, with highest expression at $24 \mathrm{~h}$ and recovery to normal levels upon repair completion (Figure 5). These data indicated that $37 L R P$ plays an important role in shell repair and mantle growth.

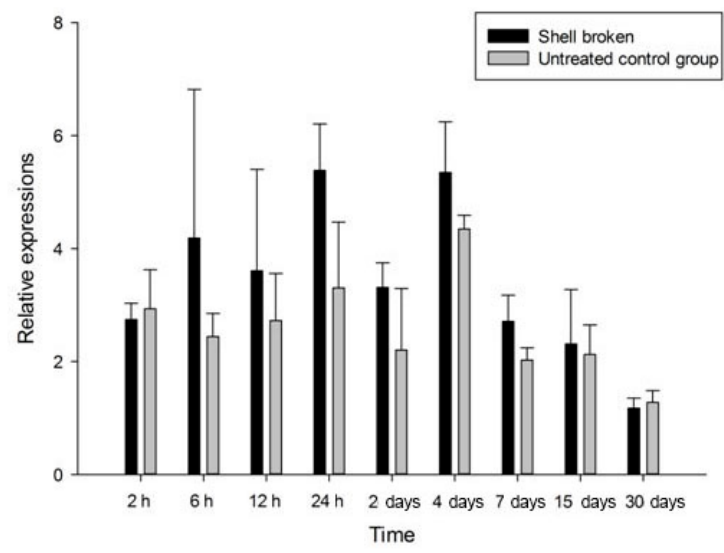

Figure 5. Relative expression of $37 L R P$ in the mantle after shell damage at each sampling time point.

\section{In situ hybridization of $37 L R P$ in the mantle}

A strong positive signal for expression of $37 L R P$ was observed in the external epithelial cells in the mantle, internal and external epithelial cells in the evagination, and in the epithelial cell layer in the peritoneum (Figure 6).

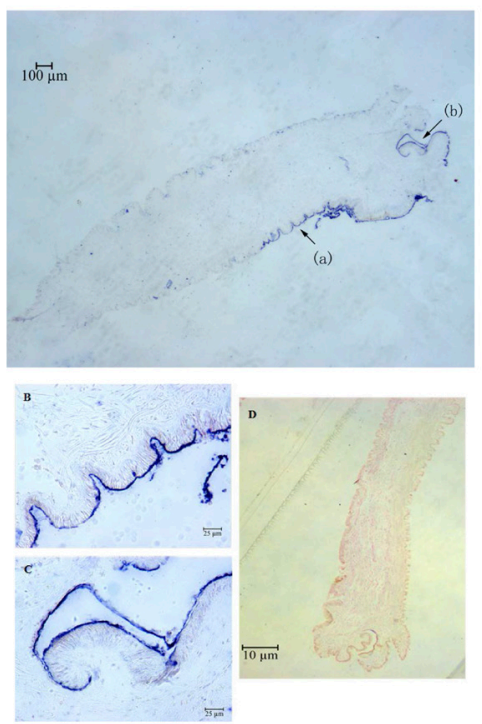

Figure 6. In situ hybridization of $37 L R P$ in tissues. A. B. C. Positive in situ hybridization. D. Negative control. B is the magnified image of the arrow (a) in A; C is the magnified image of the arrow (b) in A. Blue-violet indicates a positive hybridization signal. 


\section{DISCUSSION}

The $37-\mathrm{kDa}$ LRP is the precursor of the $67-\mathrm{kDa} \mathrm{LR}$, which is a highly conserved protein with many important functions. The cloned $37 \mathrm{LRP}$ from $\mathrm{H}$. cumingii exhibited high similarity with those of other species, such as $P$. fucata (80\%) and mammals and amphibians (over 70\%), which confirmed its high degree of conservation. Ardini et al. (1998) analyzed the relationship between $37 L R P$ and its homologous protein, ribosomal protein $\mathrm{p} 40$, and found that both had a common ancestor, although $37 L R P$ is slightly smaller than p40 and has a particular palindromic sequence, LMWWML, with the ability to combine with LN (AcostaSalmon and Southgate, 2005). The 37LRP of H. cumingii has two LN binding sites, one being designated the $\mathrm{G}$ peptide, which contains the particular palindromic sequence and binds LN with high-affinity, and another, located at the C-terminal (residues 205-229), which combines with the YIGSR sequence of the LN $\beta 1$ chain (Awaji and Suzuki, 1995; Miyamoto et al., 1996) (Figure 2). The amino acid sequences are not identical in different species, although a high degree of conservation was observed among vertebrates and slight variation was observed among invertebrates. For H. cumingii, P. fucata, and Drosophila, the palindromic sequence in the G peptide was altered from LMWWML to LMWWLL with only one amino acid residue difference. This indicates that the LN binding function of $37 L R P$ is important, and is therefore evolutionarily conserved.

qPCR results showed that $37 L R P$ was generally expressed in various tissues of $H$. cumingii, with highest expression in the liver and lowest in blood. In order to explore the influence and function of $37 L R P$ on shell and pearl formation, shell damage was inflicted in H. cumingii and $37 L R P$ expression in the mantle and liver were analyzed by qPCR. The result showed that expression of $37 L R P$ in the experimental group and the control group exhibited the same trend in the liver from the early stage of the injury to completed repair. This trend was characterized by an initial increase followed by decreased expression and a subsequent increase. The liver is a tissue associated with abundant secretions and high metabolic activity. At the early stage of injury, the liver can enhance its anabolic function and generate an immune response resulting in increased $37 L R P$ expression. The intermediate period was associated with shell repair and reduced liver metabolism, resulting in decreased 37LRP expression. Once the shell repair was complete, liver metabolism recovered to normal levels resulting with concomitant recovery of normal $37 L R P$ expression levels. In the mantle, expression of $37 L R P$ was initially increased following shell injury and then decreased. At $2 \mathrm{~h}$ post-injury, there was no difference in the expression of $37 L R P$ between the experimental and control groups. Between $6 \mathrm{~h}$ and 15 days, its expression was significantly higher in the experimental group than that in the control group. Subsequently, expression was recovered to normal levels due to basic completion of the repair of the shell and mantle, with no significant difference between the two groups. Studies have shown that the secretory capacity of the mantle is recovered following injury (Gardner et al., 2011) with development of the epithelial and glandular cells for 15 days post-injury, which was consistent with our results.

In situ hybridization studies showed that $37 L R P$ mRNA was expressed in the horny groove (Figure 6C), that is, the external epithelial cells in the central pleats and the internal epithelial cells in outer pleats. The surface of this structure secretes the polysaccharide-protein complexes with the adhesion ability to form the cuticle (Sudo et al., 1997). It was speculated that the expression of $37 L R P$ was related to cuticle formation. As shown Figure 6A [box (a) 
and arrow], 37LRP mRNA was also expressed in the external epithelial cells in the outer pleats and the border membrane, which are considered to be associated with the formation of the shell prismatic and nacreous layers (Sudo et al., 1997; Tsukamoto et al., 2004; Takeuchi and Endo, 2006; Fu et al., 2008). In situ hybridization analysis of 37LRP expression in P. fucata performed by $\mathrm{Fu}$ et al. (2008) demonstrated a strong positive signal at the edge of the mantle and in the epithelial cells of the border membrane. Expression was predominantly distributed in the flat epithelial tissue, in which cells showed stronger secretion and proliferation. The $37 L R P$ has been shown to be involved in epithelial cell proliferation and nacre secretion (Gloe et al., 1999), which is consistent with its detection in the mantle by in situ hybridization. Combined with the fluorescence quantitative results in broken shells, the 37-kDa LRP can be considered to be involved in the formation of the cuticle and the prismatic and nacreous layers.

\section{ACKNOWLEDGMENTS} (\#ZF1206).

Research supported by the Shanghai University Knowledge Service Platform

\section{REFERENCES}

Acosta-Salmon H and Southgate PC (2005). Mantle regeneration in the pearl oysters Pinctada fucata and Pinctada margaritifera. Aquaculture 246: 447-453.

Ardini E, Pesole G, Tagliabue E, Magnifico A, et al. (1998). The 67-kDa laminin receptor originated from a ribosomal protein that acquired a dual function during evolution. Mol. Biol. Evol. 15: 1017-1025.

Awaji M and Suzuki T (1995). The pattern of cell proliferation during pearl sac formation in the pearl oyster. Fish. Sci. 61: 747-751.

Bai Z, Yin Y, Hu S, Wang G, et al. (2009). Identification of genes involved in immune response, microsatellite, and SNP markers from expressed sequence tags generated from hemocytes of freshwater pearl mussel (Hyriopsis cumingii). Mar. Biotechnol. 11: 520-530.

Castronovo V, Taraboletti G and Sobel ME (1991). Functional domains of the 67-kDa laminin receptor precursor. J. Biol. Chem. 266: 20440-20446.

Coggin JH, Barsoum AL and Rohrer JW (1999). 37 Kilodalton oncofetal antigen protein and immature laminin receptor protein are identical, universal T-cell inducing immunogenes on primary rodent and human cancers. Anticancer Res. 19: 5535-5542.

Donaldson EA, McKenna DJ, McMullen CB, Scott WN, et al. (2000). The expression of membrane-associated 67-kDa laminin receptor (67LR) is modulated in vitro by cell-contact inhibition. Mol. Cell Biol. Res. Commun. 3: 53-59.

Fu YP, Xie LP and Zhang RQ (2008). Cloning and characterization of $37 \mathrm{kDa}$ laminin receptor precursor in pearl oyster, Pinctada fucata. Progr. Nat. Sci. 18: 233-238.

Gardner LD, Mills D, Wiegand A, Leavesley D, et al. (2011). Spatial analysis of biomineralization associated gene expression from the mantle organ of the pearl oyster Pinctada maxima. BMC Genomics 12: 455.

Gloe T, Riedmayr S, Sohn HY and Pohl U (1999). The 67-kDa laminin-binding protein is involved in shear stressdependent endothelial nitric-oxide synthase expression. J. Biol. Chem. 274: 15996-16002.

Kibbey MC, Grant DS and Kleinman HK (1992). Role of the SIKVAV site of laminin in promotion of angiogenesis and tumor growth: an in vivo Matrigel model. J. Natl. Cancer Inst. 84: 1633-1638.

Landowski TH, Uthayakumar S and Starkey JR (1995). Control pathways of the $67 \mathrm{kDa}$ laminin binding protein: surface expression and activity of a new ligand binding domain. Clin. Exp. Metastasis 13: 357-372.

Li XL, Wang GL, Li JL and Yuan YM (2010). Full-length cDNA cloning and encoding protein structure analysis of GPX in Hyriopsis cumingii. Yi. Chuan 32: 360-368.

Livak KJ and Schmittgen TD (2001). Analysis of relative gene expression data using real-time quantitative PCR and the 2(-Delta Delta C(T)) method. Methods 25: 402-408.

Magnifico A, Tagliabue E, Buto S, Ardini E, et al. (1996). Peptide G, containing the binding site of the 67-kDa laminin receptor, increases and stabilizes laminin binding to cancer cells. J. Biol. Chem. 271: 31179-31184. 
Malinda KM, Nomizu M, Chung M and Delgado M (1999). Identification of laminin a1 and b1 chain peptides active for endothelial cell adhesion, tube formation, and aortic sprouting. Fed. Am. Soc. Exp. Biol. J. 13: 53-62.

McKenna DJ, Simpson DA, Feeney S, Gardiner TA, et al. (2001). Expression of the $67 \mathrm{kDa}$ laminin receptor (67LR) during retinal development: correlations with angiogenesis. Exp. Eye Res. 73: 81-92.

Menard S, Castronovo V, Tagliabue E and Sobel ME (1997). New insights into the metastasis-associated $67 \mathrm{kD}$ laminin receptor. J. Cell Biochem. 67: 155-165.

Miyamoto H, Miyashita T, Okushima M, Nakano S, et al. (1996). A carbonic anhydrase from the nacreous layer in oyster pearls. Proc. Natl. Acad. Sci. U. S. A. 93: 9657-9660.

Nomizu M, Kuratomi Y, Malinda KM, Song SY, et al. (1998). Cell binding sequences in mouse laminin alpha1 chain. $J$. Biol. Chem. 273: 32491-32499.

Sanjuan X, Fernandez PL, Miquel R, Munoz J, et al. (1996). Overexpression of the 67-kD laminin receptor correlates with tumour progression in human colorectal carcinoma. J. Pathol. 179: 376-380.

Satoh K, Narumi K, Abe T, Sakai T, et al. (1999). Diminution of 37-kDa laminin binding protein expression reduces tumour formation of murine lung cancer cells. Br. J. Cancer 80: 1115-1122.

Sudo S, Fujikawa T, Nagakura T, Ohkubo T, et al. (1997). Structures of mollusc shell framework proteins. Nature 387 : 563-564.

Suzuki T, Yoshinaka R, Mizuta S and Funakoshi S (1991). Extracellular matrix formation by amebocytes during epithelial regeneration in the pearl oyster Pinctada fucata. Cell Tissue Res. 266: 75-82.

Takeuchi T and Endo K (2006). Biphasic and dually coordinated expression of the genes encoding major shell matrix proteins in the pearl oyster Pinctada fucata. Mar. Biotechnol. 8: 52-61.

Taraboletti G, Belotti D, Giavazzi R, Sobel ME, et al. (1993). Enhancement of metastatic potential of murine and human melanoma cells by laminin receptor peptide G: attachment of cancer cells to subendothelial matrix as a pathway for hematogenous metastasis. J. Natl. Cancer Inst. 85: 235-240.

Tsukamoto D, Sarashina I and Endo K (2004). Structure and expression of an unusually acidic matrix protein of pearl oyster shells. Biochem. Biophys. Res. Commun. 320: 1175-1180.

Vacca A, Ribatti D, Roncali L, Lospalluti M, et al. (1993). Melanocyte tumor progression is associated with changes in angiogenesis and expression of the 67-kilodalton laminin receptor. Cancer 72: 455-461. 